\title{
EDITORIAL
}

nature

cell biology

\section{Nature Reports Stem Cells}

\section{Nature Publishing Group will launch an online resource for stem cell biology.}

Stem cell research is only a quarter century old and it is already the most publicly visible of the biological sciences. This is not surprising, given the broad clinical potential, the ethically charged directions that some of the research is taking us in and, above all, the magic of uncovering the molecular nature of self renewal and immortality. The stem cell community has grown significantly and the field touches on most other disciplines, as stem cells have been found in a plethora of embryonic and adult tissues, and as the molecular mechanisms underlying self renewal and commitment have begun to emerge.

It is has been remarkable how proactive and successful a number of leaders in the stem cell community have been in publicly representing research interests. Nevertheless, the interdisciplinary nature of stem cell biology and the associated political and ethical complexities necessitate a dedicated international professional body to formally represent the community, as much as they require an authoritative forum to foster information exchange between researchers and to keep the public and policymakers abreast of the latest research. The foundation of the International Society for Stem Cell Research (ISSCR; http://www.isscr.org) in 2002 was one essential step. In the meantime, many journals have been actively covering stem cell biology. However, a dedicated forum that ties together basic and medical stem cell research, and that reports on ethical, political and economical aspects of the research has been missing.

With this in mind, Nature Publishing Group will launch a gateway dedicated to stem cell research in the late spring. Nature Reports Stem Cells will be a permanent publication providing news, research, comment and analysis; it will join the existing Nature Reports Avian Flu and, soon, climate change (http://www.nature. com/reports/). Dedicated editors well versed with stem cell research will provide digestible summaries of selected research papers, and also report on funding, legislative, ethical and community news. Events, jobs alerts and a key-worded library of relevant news and primary research content across the Nature titles will complement the site. A time line covering the last 25 years will chronicle progress and a 'frequently asked questions' section will serve as a primer for the general reader. The site will also experiment with publishing referee reports on selected stem cell papers. Importantly, the community will be strongly involved in shaping the content: a changing 'featured editor' will provide the site with their view of the latest developments, a blog aims to foster active debate and - similar to Nature China (see March 2007 editorial) - users are invited to nominate, comment and vote on research papers. Later on, databases will be added that will cover current stem cell lines, reagents, clinical trials and funding.

We invite you to sign up for this free service and to actively participate in shaping its content.

\section{Opening the RNAi black box}

\section{Nature journals require publication of RNAi sequences.}

It is a basic premise of scientific publishing that findings reported in a paper be reproducible. This necessitates the sharing of unique reagents, as well as the adequate documentation of the methods used. We have outlined our updated policies on sharing materials in the March 2005 editorial and last month on Nature's extended online methods. Laudably, the ISSCR (see above) recently published strict guidelines for sharing human ES cell materials and data, which will hopefully help reinvigorate this essential aspect of research culture ${ }^{\mathrm{a}}$.

One of the most frequent problems regarding experimental transparency has been the release of commercial RNAi probe sequences. Testament to this are the five addenda in the last two issues of Nature Cell Biology providing the delayed release of RNAi sequences. In our view, a researcher has to be aware of the sequence of a probe used to be certain of its specificity. A company's assurances of specificity are of limited value, as the context of the experiment may be quite different to the company's own quality assessment. A case in point is a paper that Nature Cell Biology published in 2005: when the commercial RNAi probe sequence was finally obtained after publication, it turned out that the probe was predicted to have an unexpected second target (Nature Cell Biol. 7, 311-318; 2005). Aside from the 'black box' issue, it is also not sufficient to list a catalogue number, as a company may change or discontinue a product at any time.

We understand that these reagents are often produced by small biotechs in a competitive environment and that the published sequence may facilitate copying. We also appreciate that patent protection can take too long to be effective in this fast moving field. However, if this leads to restrictions on information that undermine the scientific process, researchers ultimately should look elsewhere for their reagents. In our opinion, it is unlikely that revenue would be lost on a keenly priced product; after all many easily copied or self-manufactured laboratory products not protected by trade secrets, such as precast gels or DNA purification kits, sell well.

Undoubtedly, there can be exceptional circumstances: a genome wide RNAi library will have incurred significant R\&D costs. In a case such as this, we would only expect release of the sequences of the most relevant hits identified. There is a case to be made for certain chemical modifications to RNAi probes (for example, to reduce off-target effects) to remain proprietary until covered by patents. At present, we have not enforced the release of control probes, which remain more tightly guarded by some companies to protect 'best sellers', although the release is to be strongly encouraged. To clarify our publication policy, our 'Guide to Authors' now states "The sequences of all RNAi, antisense and morpholino probes must be included in the paper.... When an unpublished library is included...the sequences of probes central to the conclusions of the paper must be presented." (http://www.nature.com/ authors/editorial_policies/availability.html)

It is encouraging to see that more far-sighted companies are beginning to open up their sequences - in the case of Dharmacon, as a matter of course, since early spring. We encourage the community to take transparency into account when choosing their reagent provider.

${ }^{a}$ References on http://www.connotea.org/user/bpulverer/tag/materials\%20sharing 